\title{
A Different Facet
}

\section{High Intensity Pulsed Lasers}

The group of G. Mainfray at Saclay has investigated in great detail the novel effects that high intensity pulsed laser fields produce in multiphoton ionization where the atom is placed in an environment where it is able to absorb simultaneously several photons. If the laser field frequency approaches an atomic transition frequency, resonant enhancement produces such a strong coupling between the radiation and the atom that the interacting, or "dressed" atom has a new excitation spectrum: energy levels are shifted, broadened or split from their bare, unperturbed values. The Saclay group have been able to produce 15 ps high intensity single mode neodymium glass laser pulses free from intensity or frequency modulation. These clean pulses can be used to investigate coherent nonresonant and resonant multiphoton ionization, with the multimode complications, which made analysis of earlier experiments so difficult, now entirely absent. Three-photon resonantly enhanced four-photon ionization of caesium has been studied, with surprisingly good agreement between the experiment and current theoretical work which included AC Stark Shifts and power broadening of participating hyperfine structure levels. In particular, the change in the order of the nonlinearity as the laser frequency is tuned through multiphoton intermediate resonance seems now to be well understood. The role of multimode action on the multiphoton absorption has been studied exhaustively. A particularly spectacular example of multiphoton ionization of xenon was presented.

\section{Superfluorescence}

Q.H.F. Yrehen has performed some beautifully clear experiments which demonstrate the cooperative process known now as superfluorescence (SF) in caesium at infrared wavelengths. Superfluorescence is the coherent radiation produced by the cooperative decay of a system of completely inverted atoms. A pulse is detected whose emission is delayed (relative to the initial excitation), whose intensity is proportional to the square of the number of radiating atoms and whose spatial distribution exhibits a strongly shape-dependent anisotropy. The process was first predicted by Dicke 25 years ago, was theoretically investigated by a very large number of authors ten years ago and first observed by Feld and coworkers five years ago in HF emission. Feld's three-level technique for establishing an inversion has formed the basis of most later experiments using for the most part metal vapours. To see "pure" SF requires a non-degenerate level structure, with SF delay time $t_{D}$ less than any of the relaxation times $\mathrm{T}_{1}, \mathrm{~T}_{2}, \mathrm{~T}_{2}$ ' and $\mathrm{T}_{2}{ }^{\star}$, with an excitation pulse short compared with $t_{D}$ and with a small Fresnel number geometry.

All these requirements can be satisfied using dye laser excitation of Cs atoms to the $7 p$ level in a cell or atomic beam. At low densities a single pulse of SF is observed but as the density increases two pulses are produced. The SF field is coherent: two different transition frequencies can be persuaded to radiate and consequent SF quantum beats have been observed. The dependence of the process on the initial "triggering" of the emission was explored by injection of a weak SF signal field into a second inverted sample, which then emitted a pulse delayed by an amount governed by the size of the injection signal. Theoretical work on the very difficult problem of the initiation of the SF decay was discussed. From one viewpoint, using an antinormally ordered operator technique the process of coherent Bloch-vector decay is seen to be triggered by vacuum fluctuations in the distributed sample; the results agree with an alternative normally ordered operator approach which emphasises the role of the initial incoherent radiation emitted from each atom which then interacts with nearby atoms. Propagation effets have also been investigated.

Important advances have been made in the past few years in the development of the excimer and exciplex VUV laser systems. These are

now as developed technologically as dye lasers were five years ago, and have great potential, including their utilization in resonantly enhanced nonlinear processes to develop shorter wavelength sources. Potential and realized applications of VUV lasers are to atomic and molecular spectroscopy, photofragment spectroscopy using the laser as a bond-breaker, and to plasma diagnostics.

Scientific applications range from two-photon spectroscopy, to the determination of third-order susceptibilities and there are many other uses in the industrial field.

\section{Environment Probing}

A topical subject concerned the way lasers can be used for remote sensing in environmental diagnostics. Remote sensing of polluants such as $\mathrm{SO}_{2}, \mathrm{NO}, \mathrm{NO}_{2}, \mathrm{CO}, \mathrm{H}_{2} \mathrm{~S}$ from the $10^{-6}$ to the $10^{-9}$ level and the detection of particles of radius 0.1 to $10 \mu \mathrm{m}$ are of current interest. Humidity and windspeed, temperature (from Raman backscatter) and cloud heights are all measurable. Long path absorption of laser light using Beer's law and known cross sections (analogous in use to a giant spectrophotometer) is actively used to detect hydrocarbons and freons, with heterodyne detection if backscatter is small. LIDAR measurements of particle densities emitted in oil refinery smoke-stack plumes were described. The ability to distinguish different types of fuel oils (and their geographical origin e.g. Forties, Oman, etc.) is of great potential. From their different wavelength and lifetime fluorescence patterns detected from airborne LIDAR, ocean oil slicks may be measured and fingerprinted. This may be of use in detecting oil discharges caused by tankers washing-out their tanks at sea after discharge of their cargo.

Peter Knight

\section{Phase Transitions}

Phase transitions is a wide subject that has implications in many domains of physics; for example structural or magnetic phase transition, polymer transition, molecular and plastic transition, order-disorder systems, non- equilibrum phase transitions, metastable states. Problems relate to:

- dimensionality of the system, of the order parameter

- competing order parameters

- central peak artefact. 